\title{
Superficial Siderosis With Verrucous Vegetation Around a Dural Defect Confirmed by Intraoperative Ultrasonography
}

\author{
Masaaki Machino ${ }^{\text {a }}$, Shiro Imagama ${ }^{\text {a, b }}$, Kei Ando ${ }^{\text {a }}$, Kazuyoshi Kobayashia, Hiroaki Nakashima ${ }^{a}$, \\ Shunsuke Kanbara ${ }^{a}$, Sadayuki Ito ${ }^{\text {a }}$, Naoki Ishiguro ${ }^{a}$
}

\begin{abstract}
Superficial siderosis (SS) of the central nervous system (CNS) is a rare disease caused by repeated hemorrhages in the subarachnoid space. We describe surgical treatment of an SS case in which a dural defect with verrucous vegetation in the spinal canal was detected by intraoperative ultrasonography (US). After laminectomy, intraoperative US imaging confirmed verrucous vegetation and epidural fluid collection around the ventral dural defect before incision of the posterior dura mater. As intraoperative US also showed a dural defect at the ventral and right sides of the spinal cord, the posterior dural incision was made in a manner that was slightly more right lateral than standard midline. The ventral dural defect was repaired by direct sutures. After repair, intraoperative US imaging confirmed dural hole closure, removal of vegetation and resolution of the collected fluid. Dural closure was successful and symptoms partially improved despite the patient's long history of SS. Intraoperative US can help surgeons make decisions about precise dural incision before repair. Intraoperative US is useful for detecting the level of dural defect and verrucous vegetation before repair and confirming closure of the dural defect, successful removal of vegetation and resolution of fluid collection after repair.
\end{abstract}

Keywords: Superficial siderosis; Dural defect; Verrucous vegetation; Intraoperative ultrasonography; Dural closure

\section{Introduction}

Superficial siderosis (SS) of the central nervous system (CNS) is a rare and chronic disease with hemosiderin deposition in the subpial layers of the brain and spinal cord caused by repetitive

Manuscript submitted May 15, 2020, accepted May 30, 2020

Published online June 29, 2020

aDepartment of Orthopedic Surgery, Nagoya University Graduate School of Medicine, Nagoya, Japan

${ }^{b}$ Corresponding Author: Shiro Imagama, Department of Orthopedic Surgery, Nagoya University Graduate School of Medicine, 65, Tsurumai, Showa-ku, Nagoya, Aichi 466-8550, Japan. Email: imagama@med.nagoya-u.ac.jp

doi: https://doi.org/10.14740/jmc3495 subarachnoid hemorrhage [1]. The patients with SS clinically have the slow progression of sensorineural hearing loss, cerebellar ataxia, and dementia [1,2]. Causes of SS include dural defect, head or back injury, nerve root injury or brachial plexus, tumors, vascular disease such as arteriovenous malformation/fistula, and prior intradural surgery. Magnetic resonance imaging (MRI) is capable of detecting hemosiderin deposits at the surface of brain and spinal cord with high sensitivity, and it can also detect accumulated fluid in the spinal canal.

Previous studies have been focused on the relationship between SS and dural defects with fluid collection in the spinal canal $[3,4]$. They described the importance of imaging examinations for detecting dural defects.

We report the surgical treatment of a case of SS in which a dural defect with verrucous vegetation and cerebrospinal fluid (CSF) collection were detectable by intraoperative ultrasonography (US). To the best of our knowledge, this is the first reported case of SS with verrucous vegetation in the spinal canal.

\section{Case Report}

A 75-year-old woman had an 18-year history of headache, progression of gait disturbance, dysarthria and auditory disorder. At the time of admission, she was alert and oriented. Neurological examination revealed right-dominant severe hearing loss, dysarthria, and urinary disturbance. The finger-nose tests showed her incoordination. The patient also had cerebellar ataxia and mild motor loss in her lower extremities. Analysis of CSF indicated an increased red blood cell count and a low opening pressure. MRI showed low intensity areas on T2weighted images on the superficial brain and spinal cord by hemosiderin deposition. Furthermore, MRI and computed tomography (CT) myelography confirmed fluid collection in the anterior spinal canal at T1 to T4 (Fig. 1a, c, d). The constructive interference in steady state (CISS) magnetic resonance (MR) images detected a dural defect at the T1/T2 level (Fig. 1b). Surgical intervention was determined to be necessary. The spinal cord appeared discolored and yellow after laminectomy from T1 to T4 level. Verrucous vegetation and epidural fluid collection around the ventral dural defect were confirmed by intraoperative US imaging before incision of the posterior dura matter (Fig. 2a). Intraoperative US also detected ventral dural defect on the right side of spinal cord (Fig. 2b). Therefore, to allow the spinal cord to be moved forward, the operating table 


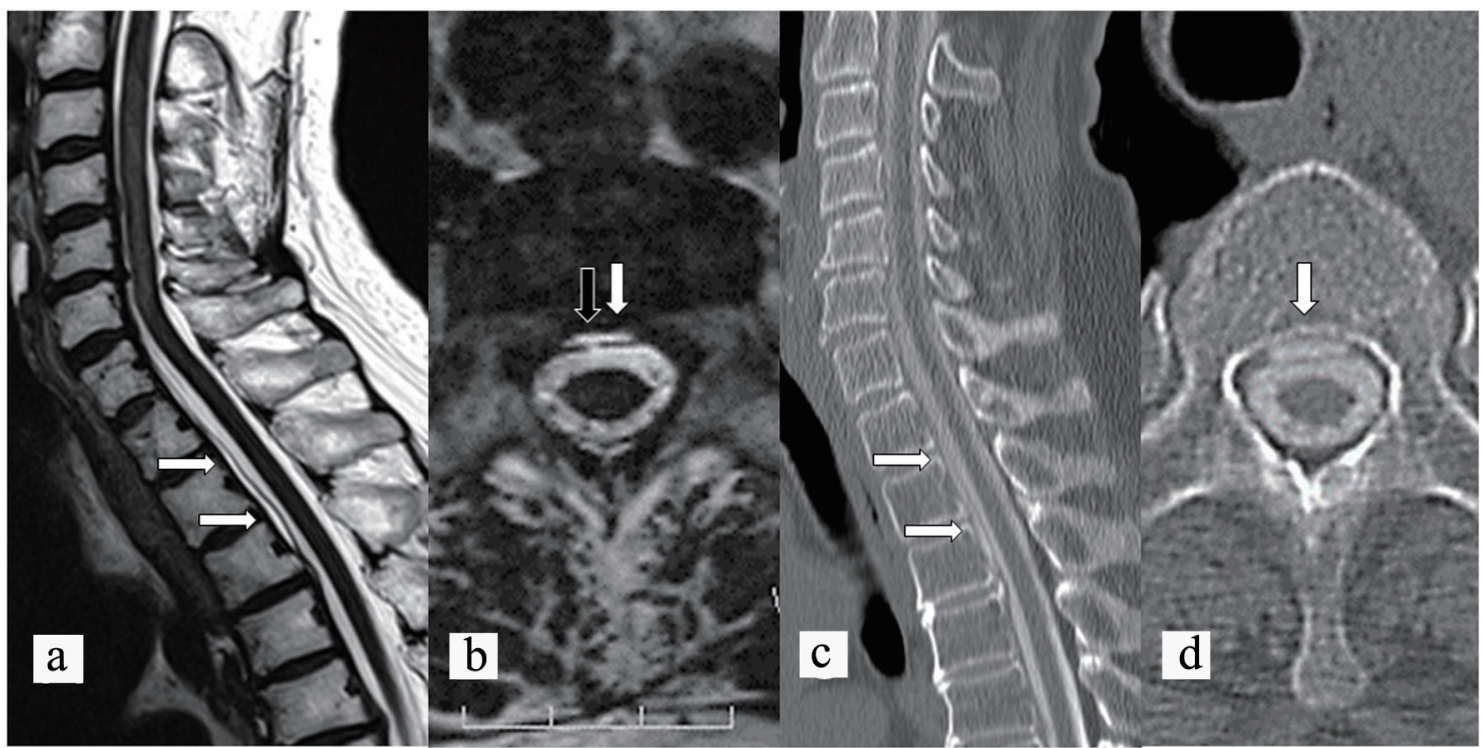

Figure 1. Preoperative sagittal (a) and axial (b) CISS MR images showing fluid collection (white arrows) in the spinal canal and a dural defect (black arrow). Preoperative sagittal (c) and axial (d) CT myelography showing longitudinal epidural CSF leakage (white arrows) ventral to the spinal cord. CISS: constructive interference in steady state; MR: magnetic resonance; CT: computed tomography; CSF: cerebrospinal fluid.

was rotated slightly to the left. Then, the posterior dural incision was made in a manner that was slightly more right lateral than standard midline. Some dentate ligaments were separated from spinal cord side as needed. With space between the spinal cord and defect, a 5-mm ventral dural defect with verrucous vegetation was confirmed on the right side near the midline at the T1/T2 level (Fig. 3a, b). The ventral dural hole was identified after removal of vegetation (Fig. 3c). A secure working space was maintained by rotation of the operating table. The dural closure was carefully performed without retraction of the spinal cord. The anterior dural tear was repaired by four direct sutures using synthetic non-absorbable monofilament surgical suture (7-0 polypropylene suture thread) and a thin needle carrier (Fig. 3d). Because the tip of the needle was narrow, it was able to operate without contacting the spinal cord. After dural closure, removal of vegetation and resolution of
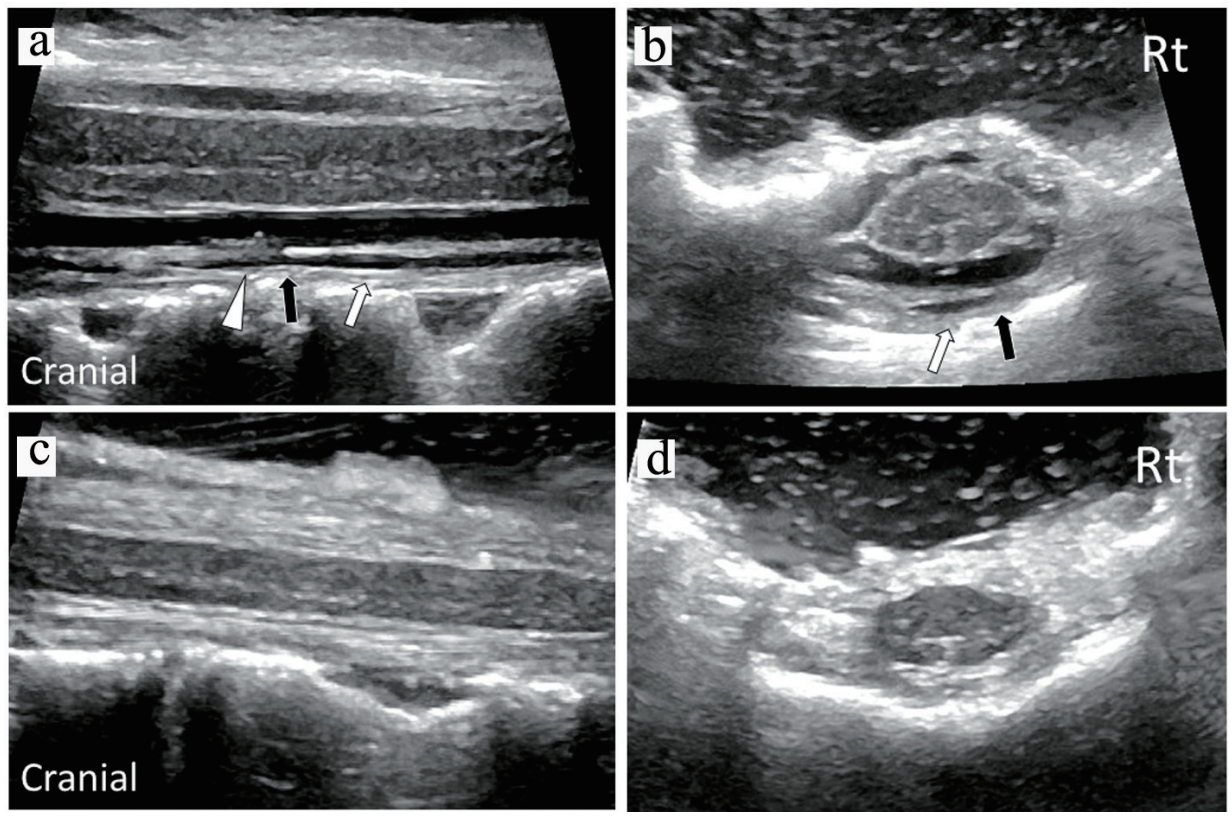

Figure 2. Intraoperative sagittal (a) and axial (b) US images confirmed verrucous vegetation (white triangle) and epidural fluid collection (white arrows) around the ventral dural defect (black arrow) before dural closure. Intraoperative sagittal (c) and axial (d) US images demonstrated removal of vegetation and resolution of fluid collection after dural closure. US: ultrasonography. 

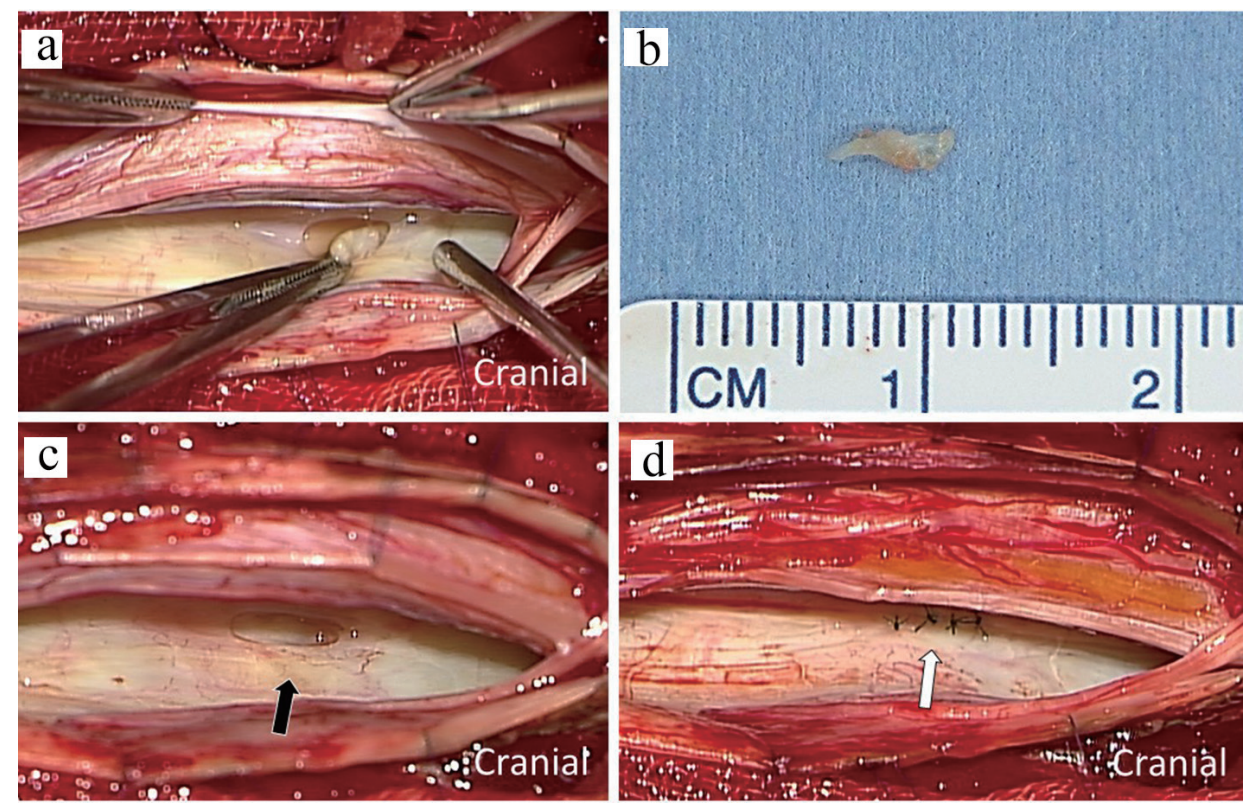

Figure 3. Intraoperative photograph showing dural defect with verrucous vegetation after incision of the posterior dura matter (a). The macroscopic image of resected specimen with scale (b). The ventral dural hole (black arrow) was detected during surgery (c). The dural defect was repaired by direct sutures (white arrows) (d).

the fluid collection were confirmed by intraoperative US images (Fig. 2c, d). Epidural fluid collection was not evident on postoperative MRI, and this status was stable during the 2-year follow-up. After the successful dural closure, the patient reported improvement in headaches, despite the long history of SS. However, the patient's ataxia, hearing loss, dysarthria, walking ability, and urinary disturbance were unchanged. The neurological symptoms were stable without deterioration.

\section{Discussion}

Repetitive subarachnoid hemorrhage sets up the hyperproduction of hemoglobin degradation products. Toxic, unbound ferric ions accumulate when the protective mechanisms are exhausted because of the chronic, recurrent hemorrhage. The subsequent free radical damage, membrane dysfunction and lipid peroxidation causing neuronal injury are suggested [5]. Currently, a relationship between repetitive hemorrhage in SS and dural abnormality in the spine has been demonstrated. Sometimes, MRI observed a longitudinal extensive fluid-filled collection in the spinal canal in SS patients. This fluid collection is associated with CSF leakage arising from dural defects in the spine. CISS MR imaging can also be used to detect defects, and the images clearly show discontinuity of the dura mater. However, it is difficult to figure out the actual location of a small dural tear even by CT myelography or thin-slice MRI in the patients with SS.

In our case, US imaging intraoperatively showed epidural collected fluid around the ventral dural defect with verrucous vegetation before incision of the posterior dura mater. By clearly showing the small dural hole, intraoperative US could help surgeon in decision making about precise dural incision before repairment. It is important to maintain a secure working space by rotating the operating table. Moderate rotation is also beneficial because the surgeon can operate on the ventral site of the spinal cord without putting a burden on it.

A repairment of dural defect has been shown to stabilize CSF leakage and subarachnoid hemorrhage regardless of the closing method. These examination findings do not dramatically improve the symptoms of SS. The successful repairment of a dural defect can halt the neurological symptom progression and might improve symptom related to CSF hypovolemia. Recently, surgical closure for the defect is the most responsible method useful to halt the progression of SS symptoms related to dural defect. In our case, the ventral fluid collection disappeared postoperatively, suggesting that the dural defect was successfully repaired. The continuous exposure of neural tissue to toxic hemosiderin deposits and free radical damage might result in irreversible and even progressive neurological disturbances. Consistent with previous studies, surgical closure of the dural defect in our case halted disease progression. Although closure might improve some symptoms related to CSF hypovolemia, its effect might be reduced in patients with longer history of SS. Thereby, earlier diagnosis, detection of the dural defect level, and surgical closure are important for keeping to minimum neurological disorder in SS patients.

\section{Conclusions}

The progression of neurological impairment in SS related to dural defect can be stopped by surgical closure. US is intraoperatively informative for detection of the dural defect site and verrucous vegetation before repairment and identifying the dural defect closure, successful removal of vegetation and 
resolution of fluid collection after repair.

\section{Acknowledgments}

None to declare.

\section{Financial Disclosure}

None to declare.

\section{Conflict of Interest}

None to declare.

\section{Informed Consent}

The patient agreed to provide her information for the publication of this case report.

\section{Author Contributions}

MM and SI participated in the literature search and drafted the manuscript. KA, KK and NI participated in the notes review. $\mathrm{HN}, \mathrm{SK}$ and SI conceived the study and participated in its de- sign and coordination and helped to draft the manuscript. All authors read and approved the final manuscript.

\section{Data Availability}

The data supporting the findings of this study are available from the corresponding author upon reasonable request.

\section{References}

1. Fearnley JM, Stevens JM, Rudge P. Superficial siderosis of the central nervous system. Brain. 1995;118(Pt 4):1051-1066.

2. Levy M, Turtzo C, Llinas RH. Superficial siderosis: a case report and review of the literature. Nat Clin Pract Neurol. 2007;3(1):54-58; quiz 59.

3. Ikeda T, Noto D, Noguchi-Shinohara M, Ono K, Takahashi $\mathrm{K}$, Ishida C, Yoshita M, et al. CSF tau protein is a useful marker for effective treatment of superficial siderosis of the central nervous system: two case reports. Clin Neurol Neurosurg. 2010;112(1):62-64.

4. Miliaras G, Bostantjopoulou S, Argyropoulou M, Kyritsis A, Polyzoidis K. Superficial siderosis of the CNS: report of three cases and review of the literature. Clin Neurol Neurosurg. 2006;108(5):499-502.

5. Koeppen AH, Dickson AC, Chu RC, Thach RE. The pathogenesis of superficial siderosis of the central nervous system. Ann Neurol. 1993;34(5):646-653. 